\title{
UPPER BOUNDS FOR THE DERIVATIVE OF EXPONENTIAL SUMS
}

\section{PETER BORWEIN AND TAMÁS ERDÉLYI}

(Communicated by Andrew Bruckner)

Abstract. The equality

$$
\sup _{p} \frac{\left|p^{\prime}(a)\right|}{\|p\|_{[a, b]}}=\frac{2 n^{2}}{b-a}
$$

is shown, where the supremum is taken for all exponential sums $p$ of the form

$$
p(t)=a_{0}+\sum_{j=1}^{n} a_{j} e^{\lambda_{j} t}, \quad a_{j} \in \mathbf{R},
$$

with nonnegative exponents $\lambda_{j}$. The inequalities

$$
\left\|p^{\prime}\right\|_{[a+\delta, b-\delta]} \leq 4(n+2)^{3} \delta^{-1}\|p\|_{[a, b]}
$$

and

$$
\left\|p^{\prime}\right\|_{[a+\delta, b-\delta]} \leq 4 \sqrt{2}(n+2)^{3} \delta^{-3 / 2}\|p\|_{L_{2}[a, b]}
$$

are also proved for all exponential sums of the above form with arbitrary real exponents. These results improve inequalities of Lorentz and Schmidt and partially answer a question of Lorentz.

\section{INTRODUCTION AND NOTATION}

Let $\Lambda_{n}:=\left\{\lambda_{1}<\lambda_{2}<\cdots<\lambda_{n}\right\}, \lambda_{j} \neq 0, j=1,2 \ldots, n$;

$$
E\left(\Lambda_{n}\right):=\left\{f: f(t)=a_{0}+\sum_{j=1}^{n} a_{j} e^{\lambda_{j} t}, a_{j} \in \mathbf{R}\right\}
$$

and

$$
E_{n}:=\bigcup_{\Lambda_{n}} E\left(\Lambda_{n}\right)=\left\{f: f(t)=a_{0}+\sum_{i=1}^{n} a_{j} e^{\lambda_{j} t}, a_{j}, \lambda_{j} \in \mathbf{R}\right\} .
$$

We will use the norms

$$
\|f\|_{[a, b]}:=\max _{x \in[a, b]}|f(x)|
$$

Received by the editors March 10, 1993 and, in revised form, August 18, 1993.

1991 Mathematics Subject Classification. Primary 41A17. 
and

$$
\|f\|_{L_{2}[a, b]}:=\left(\int_{a}^{b}|f(x)|^{2} d x\right)^{1 / 2}
$$

for functions $f \in C[a, b]$.

Schmidt [3] proved that there is a constant $c(n)$ depending on $n$ so that

$$
\left\|p^{\prime}\right\|_{[a+\delta, b-\delta]} \leq c(n) \delta^{-1}\|p\|_{[a, b]}
$$

for every $p \in E_{n}$ and $\delta \in(0,(b-a) / 2)$. Lorentz [2] improved Schmidt's result by showing that for every $\alpha>\frac{1}{2}$ there is a constant $c(\alpha)$ depending only on $\alpha$ so that $c(n)$ in the above inequality can be replaced by $c(\alpha) n^{\alpha \log n}$, and he speculated that there may be an absolute constant $c$ so that Schmidt's inequality holds with $c(n)=c n .{ }^{1}$ Theorem 2 of this paper shows that Schmidt's inequality holds with $c(n)=4(n+2)^{3}$. Our first theorem establishes the sharp inequality

$$
\left|p^{\prime}(a)\right| \leq \frac{2 n^{2}}{b-a}\|p\|_{[a, b]}
$$

for every $p \in E_{n}$ with nonnegative exponents $\lambda_{j}$.

\section{NeW RESUlts}

Theorem 1. We have

$$
\sup _{p} \frac{\left|p^{\prime}(a)\right|}{\|p\|_{[a, b]}}=\frac{2 n^{2}}{b-a}
$$

for every $a<b$, where the supremum is taken for all exponential sums $p \in E_{n}$ with nonnegative exponents. The equality

$$
\sup _{p} \frac{\left|p^{\prime}(a)\right|}{\|p\|_{[a, b]}}=\frac{2 n^{2}}{a(\log b-\log a)}
$$

also holds for every $0<a<b$, where the supremum is taken for all Müntz polynomials of the form

$$
p(x)=a_{0}+\sum_{j=1}^{n} a_{j} x^{\lambda_{j}}, \quad a_{j} \in \mathbf{R}, \lambda_{j} \geq 0 .
$$

Theorem 2. The inequalities

$$
\left\|p^{\prime}\right\|_{[a+\delta, b-\delta]} \leq 4(n+2)^{3} \delta^{-1}\|p\|_{[a, b]}
$$

and

$$
\left\|p^{\prime}\right\|_{[a+\delta, b-\delta]} \leq 4 \sqrt{2}(n+2)^{3} \delta^{-3 / 2}\|p\|_{L_{2}[a, b]}
$$

hold for every $p \in E_{n}$ and $\delta \in(0,(b-a) / 2)$.

\section{Proofs}

To prove Theorem 1 we need some notation. If $\Lambda_{n}:=\left\{\lambda_{1}<\lambda_{2}<\cdots<\lambda_{n}\right\}$ is a set of positive real numbers, then the real span of

$$
\left\{1, x^{\lambda_{1}}, x^{\lambda_{2}}, \ldots, x^{\lambda_{n}}\right\}, \quad x \geq 0,
$$

\footnotetext{
${ }^{1}$ (Added in proof) We can now prove this with $c=2$; the proof will appear elsewhere.
} 
will be denoted by $M\left(\Lambda_{n}\right)$. It is well known that these are Chebyshev spaces on $[0, \infty)$ (see [1] for instance), so $M\left(\Lambda_{n}\right)$ possesses a unique Chebyshev "polynomial" $T_{\Lambda_{n}}$ on $[a, b], 0<a<b$, with the properties

(i) $T_{\Lambda_{n}} \in M\left(\Lambda_{n}\right)$,

(ii) $\left\|T_{\Lambda_{n}}\right\|_{[a, b]}=1$, and

(iii) there are $a=x_{0}<x_{1}<\cdots<x_{n}=b$ so that

$$
T_{\Lambda_{n}}\left(x_{j}\right)=(-1)^{n-j}, \quad j=0,1, \ldots, n .
$$

It is routine to prove (see [1] again) that $T_{\Lambda_{n}}$ has exactly $n$ distinct zeros on $(a, b)$,

$$
\max _{0 \neq p \in M\left(\Lambda_{n}\right)} \frac{\left|p^{\prime}(a)\right|}{\|p\|_{[a, b]}}=\frac{\left|T_{\Lambda_{n}}^{\prime}(a)\right|}{\left\|T_{\Lambda_{n}}\right\|_{[a, b]}}=\left|T_{\Lambda_{n}}^{\prime}(a)\right|,
$$

and

$$
\max _{0 \neq p \in M\left(\Lambda_{n}\right)} \frac{|p(0)|}{\|p\|_{[a, b]}}=\frac{\left|T_{\Lambda_{n}}(0)\right|}{\left\|T_{\Lambda_{n}}\right\|_{[a, b]}}=\left|T_{\Lambda_{n}}(0)\right| .
$$

Lemma 3. Let

$$
\Lambda_{n}:=\left\{\lambda_{1}<\lambda_{2}<\cdots<\lambda_{n}\right\} \text { and } \Gamma_{n}:=\left\{\gamma_{1}<\gamma_{2}<\cdots<\gamma_{n}\right\}
$$

so that $0<\lambda_{j} \leq \gamma_{j}$ for each $j=1,2, \ldots, n$. Then

$$
\left|T_{\Gamma_{n}}^{\prime}(a)\right| \leq\left|T_{\Lambda_{n}}^{\prime}(a)\right| \text {. }
$$

Proof. Without loss of generality we may assume that there is an index $m$, $1 \leq m \leq n$, so that $\lambda_{m}<\gamma_{m}$ and $\lambda_{j}=\gamma_{j}$ if $j \neq m$, since repeated applications of the result in this situation give the lemma in the general case. First we show that

$$
\left|T_{\Gamma_{n}}(0)\right|<\left|T_{\Lambda_{n}}(0)\right| .
$$

Indeed, let $R_{\Gamma_{n}} \in M\left(\Gamma_{n}\right)$ interpolate $T_{\Lambda_{n}}$ at the zeros of $T_{\Lambda_{n}}$ and be normalized so that $R_{\Gamma_{n}}(0)=T_{\Lambda_{n}}(0)$. Then the Improvement Theorem of Pinkus and Smith [4, Theorem 2] yields

$$
\left|R_{\Gamma_{n}}(x)\right| \leq\left|T_{\Lambda_{n}}(x)\right| \leq 1, \quad x \in[a, b] .
$$

Hence, using (2) with $\Lambda_{n}$ replaced by $\Gamma_{n}$, we obtain

$$
\left|T_{\Lambda_{n}}(0)\right|=\left|R_{\Gamma_{n}}(0)\right| \leq\left|T_{\Gamma_{n}}(0)\right|,
$$

which proves (4). Using the defining properties of $T_{\Lambda_{n}}$ and $T_{\Gamma_{n}}$, we can deduce that $T_{\Lambda_{n}}-T_{\Gamma_{n}}$ has at least $n+1$ zeros in $[a, b]$ (we count every internal zero without sign change twice). Now assume that (3) does not hold; then

$$
\left|T_{\Lambda_{n}}^{\prime}(a)\right|>\left|T_{\Gamma_{n}}^{\prime}(a)\right| \text {. }
$$

This, together with (4), implies that $T_{\Lambda_{n}}-T_{\Gamma_{n}}$ has at least one zero in $(0, a)$. Hence $T_{\Lambda_{n}}-T_{\Gamma_{n}}$ has at least $n+2$ zeros in $(0, b]$. This is a contradiction, since

$$
T_{\Lambda_{n}}-T_{\Gamma_{n}} \in \operatorname{span}\left\{1, x^{\lambda_{1}}, x^{\lambda_{2}}, \ldots, x^{\lambda_{n}}, x^{\gamma_{m}}\right\}
$$

and every function from the above span can have only at most $n+1$ zeros in $(0, \infty)($ see $[3])$. 
Proof of Theorem 1. It is sufficient to prove only the second statement of the theorem, the first one can be obtained by the change of variable $x=e^{t}$. We obtain from (1) and Lemma 3 that

$$
\frac{\left|p^{\prime}(a)\right|}{\|p\|_{[a, b]}} \leq \lim _{\delta \rightarrow 0+} \frac{\left|T_{\Lambda_{n, \delta}}^{\prime}(a)\right|}{\left\|T_{\Lambda_{n, \delta}}\right\|_{[a, b]}}=\lim _{\delta \rightarrow 0+}\left|T_{\Lambda_{n, \delta}}^{\prime}(a)\right|
$$

for every $p$ of the form

$$
p(x)=a_{0}+\sum_{j=1}^{n} a_{j} x^{\lambda_{j}}, \quad a_{j} \in \mathbf{R}, \lambda_{j}>0,
$$

where

$$
\Lambda_{n, \delta}:=\{\delta, 2 \delta, 3 \delta, \ldots, n \delta\}
$$

and $T_{n, \delta}$ is the Chebyshev "polynomial" of $M\left(\Lambda_{n, \delta}\right)$ on $[a, b]$. From the definition and uniqueness of $T_{\Lambda_{n, \delta}}$ it follows that

$$
T_{\Lambda_{n, \delta}}(x)=T_{n}\left(\frac{2}{b^{\delta}-a^{\delta}} x^{\delta}-\frac{b^{\delta}+a^{\delta}}{b^{\delta}-a^{\delta}}\right),
$$

where $T_{n}(y):=\cos (n \arccos y)$. Therefore,

$$
\begin{aligned}
\left|T_{\Lambda_{n, \delta}}^{\prime}(a)\right| & =\left|T_{n}^{\prime}(-1)\right| \frac{2}{b^{\delta}-a^{\delta}} \delta a^{\delta-1} \\
& =\frac{2 n^{2}}{\delta^{-1}\left(b^{\delta}-1\right)-\delta^{-1}\left(a^{\delta}-1\right)} a^{\delta-1} \underset{\delta \rightarrow 0+}{\longrightarrow} \frac{2 n^{2}}{a(\log b-\log a)}
\end{aligned}
$$

and the theorem is proved.

To prove Theorem 2 we need two lemmas.

Lemma 4. For every set $\Lambda_{n}:=\left\{\lambda_{1}<\lambda_{2}<\cdots<\lambda_{n}\right\}$ of nonzero real numbers there is a point $y \in[-1,1]$ depending only on $\Lambda_{n}$ so that

$$
\left|p^{\prime}(y)\right| \leq 2(n+2)^{3}|| p \|_{L_{2}[-1,1]}
$$

for every $p \in E\left(\Lambda_{n}\right)$.

Proof. Take the orthonormal set $\left\{p_{k}\right\}_{k=0}^{n}$ on $[-1,1]$ defined by

(i) $p_{k} \in \operatorname{span}\left\{1, e^{\lambda_{1} t}, e^{\lambda_{2} t}, \ldots, e^{\lambda_{k} t}\right\}, k=0,1, \ldots, n$;

(ii) $\int_{-1}^{1} p_{i} p_{j}=\delta_{i, j}, 0 \leq i \leq j \leq n$.

Writing $p \in E\left(\Lambda_{n}\right)$ as a linear combination of the functions $p_{k}, k=0,1, \ldots$, $n$, and using the Cauchy-Schwartz inequality and the orthonormality of $\left\{p_{k}\right\}_{k=0}^{n}$ on $[-1,1]$, we obtain in a standard fashion that

$$
\max _{p \in E\left(\Lambda_{n}\right)} \frac{\left|p^{\prime}\left(t_{0}\right)\right|}{\|p\|_{L_{2}[-1,1]}}=\left(\sum_{k=0}^{n} p_{k}^{\prime}\left(t_{0}\right)^{2}\right)^{1 / 2}, \quad t_{0} \in \mathbf{R} .
$$

Let

$$
A_{k}:=\left\{t \in[-1,1]:\left|p_{k}(t)\right| \geq(n+1)^{1 / 2}\right\}, \quad k=0,1, \ldots, n,
$$

and

$$
B_{k}:=\left\{t \in[-1,1] \backslash A_{k}:\left|p_{k}^{\prime}(t)\right| \geq 2(n+2)^{5 / 2}\right\}, \quad k=0,1, \ldots, n .
$$


Since $\int_{-1}^{1} p_{k}^{2}=1$, we have

$$
m\left(A_{k}\right) \leq(n+1)^{-1}, \quad k=0,1, \ldots, n .
$$

Since $\operatorname{span}\left\{1, e^{\lambda_{1} t}, e^{\lambda_{2} t}, \ldots, e^{\lambda_{k} t}\right\}$ is a Chebyshev system, each $\tilde{A}_{k}:=[-1,1] \backslash$ $A_{k}$ comprises of at most $k+1$ intervals and each $B_{k}$ comprises of at most $2(k+1)$ intervals. Therefore,

$$
2(n+2)^{5 / 2} m\left(B_{k}\right) \leq \int_{B_{k}}\left|p_{k}^{\prime}(t)\right| d t \leq 4(k+1) \sqrt{n+1},
$$

whence

$$
\sum_{k=0}^{n} m\left(B_{k}\right) \leq \frac{2 \sqrt{n+1}}{(n+2)^{5 / 2}} \frac{(n+1)(n+2)}{2}<1
$$

Now let

$$
A:=[-1,1] \backslash \bigcup_{k=0}^{n}\left(A_{k} \cup B_{k}\right)
$$

Then

$$
\begin{aligned}
m(A) & \geq 2-\sum_{k=0}^{n} m\left(A_{k}\right)-\sum_{k=0}^{n} m\left(B_{k}\right) \\
& >2-(n+1)(n+1)^{-1}-1=0,
\end{aligned}
$$

so there is a point $y \in A \subset[-1,1]$ where

$$
\left|p^{\prime}(y)\right| \leq 2(n+1)^{5 / 2}, \quad k=0,1, \ldots, n .
$$

Hence,

$$
\left(\sum_{k=0}^{n} p_{k}^{\prime}(y)^{2}\right)^{1 / 2} \leq 2(n+2)^{3}
$$

and the lemma is proved.

Lemma 5. We have

$$
\left|p^{\prime}(0)\right| \leq 2(n+2)^{3}\|p\|_{L_{2}[-2,2]} \leq 2(n+2)^{3}\|p\|_{[-2,2]}
$$

for every $p \in E_{n}$.

Proof. Let $\Lambda_{n}:=\left\{\lambda_{1}<\lambda_{2}<\cdots<\lambda_{n}\right\}$ be a fixed set of nonzero real numbers, and let $y \in[-1,1]$ be chosen by Lemma 4 . Let $0 \not \equiv p \in E\left(\Lambda_{n}\right)$. Then

$$
q(t):=p(t-y) \in E\left(\Lambda_{n}\right)
$$

therefore, applying Lemma 4 to $q$, we obtain

$$
\frac{\left|p^{\prime}(0)\right|}{\|p\|_{L_{2}[-2,2]}} \leq \frac{\left|p^{\prime}(0)\right|}{\|p\|_{L_{2}[-1-y, 1-y]}}=\frac{\left|q^{\prime}(y)\right|}{\|q\|_{L_{2}[-1,1]}} \leq 2(n+2)^{3},
$$

and the lemma is proved.

Proof of Theorem 2. Let $t_{0} \in[a+\delta, b-\delta]$. Applying Lemma 5 to $q(t):=$ $p\left(\delta t / 2+t_{0}\right)$, we get the theorem. 


\section{REFERENCES}

1. S. Karlin and W. J. Studden, Tchebycheff systems: with applications in analysis and statistics, Interscience, New York, 1966.

2. G. G. Lorentz, Notes on approximation, J. Approx. Theory 56 (1989), 360-365.

3. E. Schmidt, Zur Kompaktheit der Exponentialsummen, J. Approx. Theory 3 (1970), 445459.

4. P. W. Smith, An improvement theorem for Descartes systems, Proc. Amer. Math. Soc. 70 (1978), 26-30.

Department of Mathematics and Statistics, Simon Fraser University, Burnaby, British Columbia, CANADA V5A IS6

E-mail address: PBORWEINCCECM.SFU.CA

$E$-mail address: ERDELYIOCS.SFU.CA 